Pankiv M. V., Paltov Y. V. Pathomorphological changes in the structure of intervertebral disc after seven and fourteen days of experimental opioid use. Journal of Education, Health and Sport. 2020;10(10):116-126. eISSN 2391-8306. DOI http://dx.doi.org/10.12775/JEHS.2020.10.10.011

https://apcz.umk.pl/czasopisma/index.php/JEHS/article/view/JEHS.2020.10.10.011

https://zenodo.org/record/4247966

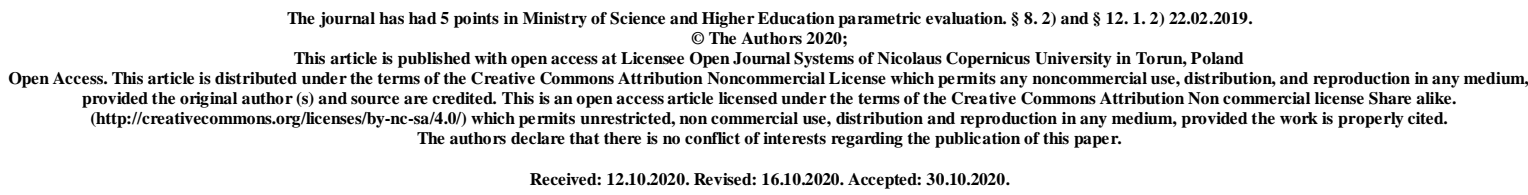

UDK 611.711.018.3/.4:615.214.24].08

\title{
Pathomorphological changes in the structure of intervertebral disc after seven and fourteen days of experimental opioid use
}

\author{
M.V. Pankiv, Y.V. Paltov \\ Danylo Halytsky Lviv National Medical University, \\ Department of Normal Anatomy, Lviv, Ukraine
}

Pankiv M. https://orcid.org/0000-0002-3714-2577

Paltov Y https://orcid.org/0000-0002-2622-4753

\section{Abstract}

We aimed to study the pathomorphological changes in the structure of intervertebral disc in the early stages of the experimental opioid use. This goal was achieved by the use of histological visualization of the intervertebral disc's structures. Histological specimens were prepared according to the typical methods using such specimens as hematoxylin, eosin, azan and due to Heidenhain method, McManus PAS-reaction and Steedman alcian blue method.

The results of this study will let form a pathomorphological basis that could be used for comparative characterization of the growth dynamics of pathomorphological changes in the intervertebral disc's structures in the early stages and compare these changes to pathomorphological signs in the late stages of experimental opioid use. These findings will provide an opportunity to establish the most appropriate time limits when these pathomorphological changes in the structure of the intervertebral disc develop and to observe the dynamics of these changes in the long term experimental opioid use. 


\section{Keywords: opioid, intervertebral disc; rat; early terms.}

Introduction. In recent years, musculoskeletal pathologies, especially spine diseases, have taken the leading position in the causes of human disability. A significant percentage of vertebrological pathologies - scoliosis, osteochondrosis, intervertebral hernias, etc., arise as a result of exposure of various factors (hypodynamic, inadequate exercises, metabolic disorders) and manifest by changes of various degrees in the intervertebral discs. [1, 3-5].

It is obvious that early detection of such changes before the onset of clinical signs of the disease will help to provide its early treatment and prevent from the development of pathological process. Thus, it is important to search for diagnostic methods of patients' examination, which would identify the signs of pathology in the preclinical stages, and to create the criteria for early diagnosis, which requires a deep and flawless study of spinal structures without pathology, taking into account gender, constitutional and individual features $[2,6-8]$.

Considering all abovementioned we believe that this study is relevant.

Materials and methods. The objects of the study were 32 mature, outbred male rats, weight - $92 \mathrm{~g}$, age - 4.5 months. Animals had been injected with nalbuphine intramuscularly once per day (at 10-11 a.m.) for 14 days. The initial dose of nalbuphine was $8 \mathrm{mg} / \mathrm{kg}$ on the first day and $15 \mathrm{mg} / \mathrm{kg}$ on the second day. Thus, conditions for chronic opioid's exposure were created [9].

Animals were divided into 2 groups. 1st group was injected with nalbuphine for 14 days (at 10-11 a.m.) with following histological materials sampling at the end of the first and second week of experimental opioid use; control group was injected with saline at 10-11 a.m. for 14 days.

All animals were kept in a vivarium, the experiment was conducted in accordance with the principles of bioethics and The European Convention for the Protection of Vertebrate Animals used for Experimental and other Scientific (Strasbourg, 1986), Council Directive 86/609 / EEC (1986), The Law of Ukraine № 3447-IV "On protection of animals from brutal treatment", general ethical principles of animal experiments, approved by the First National Congress of Ukraine on Bioethics (2001), confirmed by the conclusion of members of the Commission on Bioethics of Danylo Halytsky Lviv National Medical University (protocol №10, December 15 $5^{\text {th }}, 2019$ ).

Before sampling, the animals were withdrawn from experiment using dibutyl ether. Intervertebral discs of rats with preserved topographic ratio of the structures were used as a 
material for microstructural investigaton. Histological sections had a thickness 5 - $7 \mu \mathrm{m}$. Histological specimens were prepared according to the typical methods using hematoxylin, eosin, azan and due to Heidenhain method, McManus PAS-reaction and Steedman Alcian blue method [10,11]. Microscopic investigations and specimens photography were performed using microscope MBI-1 and Nicon D3100 camera.

\section{Results}

After 7 days of opioid injections at a dose of $8 \mathrm{mg} / \mathrm{kg}$ we found the initial changes in the structure of intervertebral disc such as moderate degenerative alterations of notochordal cells in nucleus pulposus and collagen fibers dissection in annulus fibrosus.

Nucleus pulposus was slightly reduced (Figures. 1, 2) and lost its ellipsoidal elongated shape.

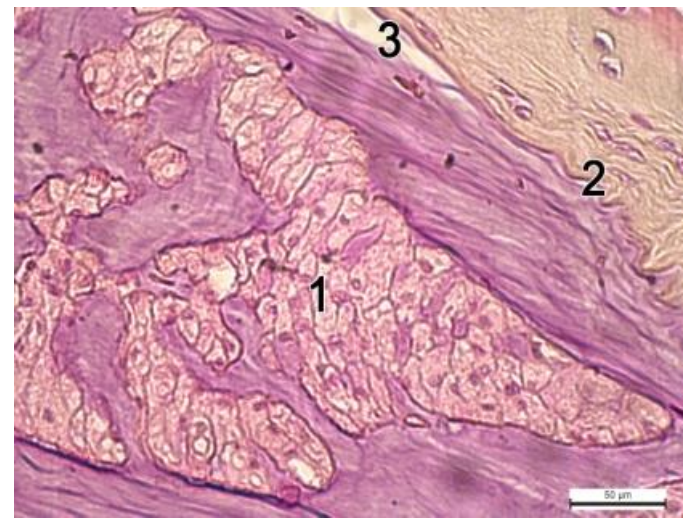

Figure. 1. Intervertebral disc after 7 days of opioid use. Hematoxylin and eosin staining. Photomicrograph x 400 .

1 - volume decrease of the nucleus pulposus;

2- chaotically arranged collagen bundles;

3 - local dissection of annulus fibrosus collagen fibers from cartilage endplate.

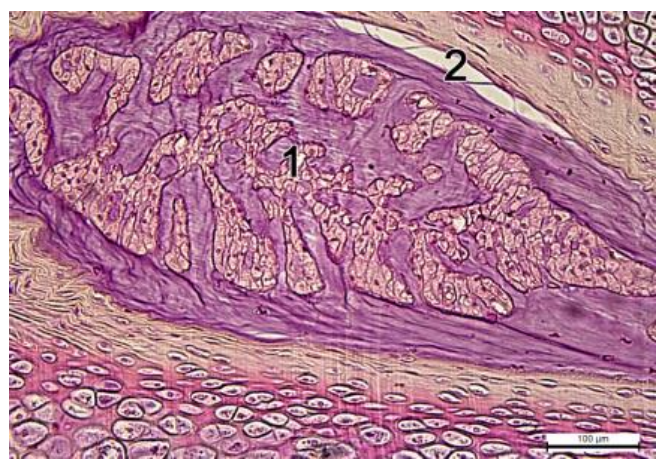

Figure 2. Intervertebral disc after 7 days of opioid use. Hematoxylin and eosin staining. Photomicrograph x 200 .

1 - volume decrease of the nucleus pulposus;

2- local dissection of annulus fibrosus collagen fibers from cartilage endplate. 
Most notochordal cells were preserved, forming syncytium. In some areas of the nucleus pulposus, single, isolated notochordal cells were visualized, the cytoplasm of which was swollen, somewhere illuminated, and inhomogeneously colored. The annulus fibrosus mostly retained its shape. Some collagen bundles swelled, increased slightly in volume, part of them shifted significantly in the area of the nucleus pulposus. Some collagen (fibrous) bundles dissected from the cartilage endplate (Figure 3).

Collagen fibers of the outer zone of annulus fibrosus were arranged chaotically (Figure 4), dissected, and between them the longitudinal cracks were visualized (Figure 5). In these areas slits filled with optically light masses were formed. The density of fibrochondrocytes was slightly decreased.

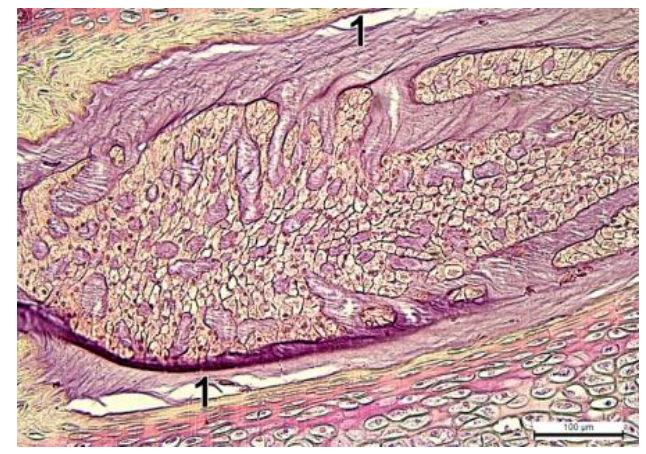

Figure 3. Intervertebral disc after 7 days of opioid use. Hematoxylin and eosin staining. Photomicrograph x 200.

1- local dissection of collagen fibers in annulus fibrosus from cartilage endplate.

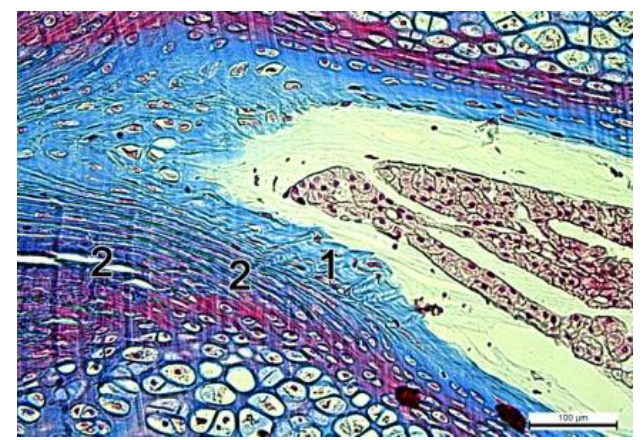

Figure 4. Intervertebral disc after 7 days of opioid use. Azan staining. Photomicrograph $x$ 200.

1- chaotically arranged collagen fibers of annulus fibrosus;

2- small longitudinal cracks in the outer zone of the annulus fibrosus. 


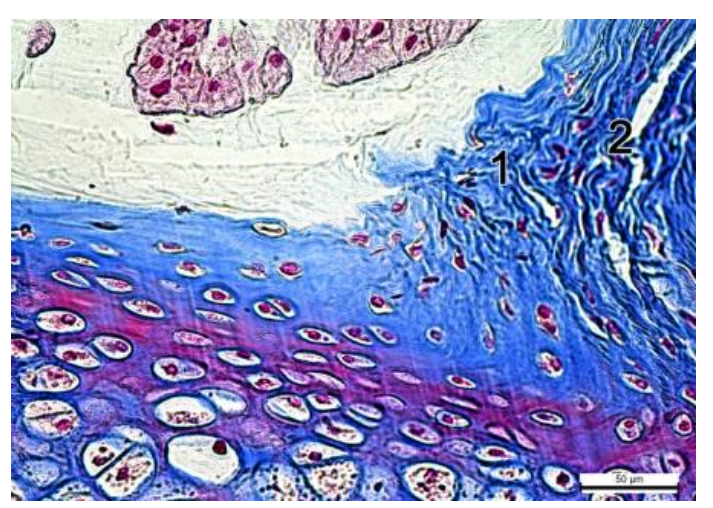

Figure 5. Intervertebral disc after 7 days of opioid use. Azan staining. Photomicrograph $x$ 400.

1- $\quad$ uneven swelling and dissection of collagen fibers of the annulus fibrosus;

2- cracks between collagen fibers.

The development of dystrophic changes accompanied by swelling, enlightenment and vacuolation of the cytoplasm was found in the chondrocytes of cartilage endplates. Insignificant proliferation of epiphyseal cartilage chondrocytes was noted; it was accompanied by dystrophic changes of cells in isogenic groups. The cytoplasm of chondrocytes was vacuolated. A significant amount of secretion accumulated in the pericellular zone of some chondrocytes.

During the PAS reaction, a moderate decrease in the color intensity of the main substance of the nucleus pulposus matrix was found, which indicates a decrease in the content of primary glycosaminoglycans (Figures 6, 7). The granules of acidic glycosaminoglycans were located inhomogeneously, their content in most parts of the nucleus pulposus was decreased (Figures 8, 9).

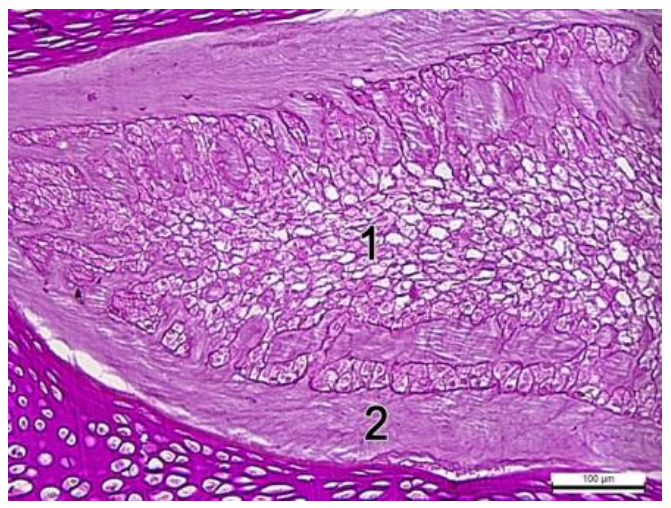

Figure 6. Intervertebral disc after 7 days of opioid use. Staining: PAS-reaction after to McManus. Photomicrograph x 200.

1- decrease in the content of glycosaminoglycans in the nucleus pulposus;

2- decrease in the content of glycosaminoglycans in the annulus fibrosus. 


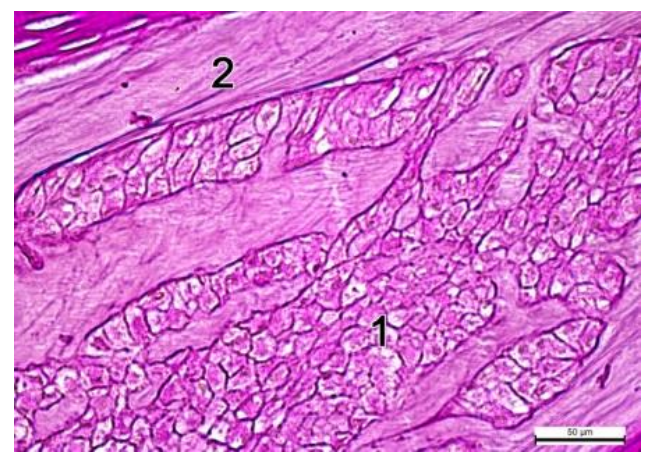

Figure 7. Intervertebral disc after 7 days of opioid use. Staining: PAS-reaction after McManus. Photomicrograph x 400.

1- decrease in the content of primary glycosaminoglycans in the nucleus pulposus;

2- decrease in the content of primary glycosaminoglycans in the annulus fibrosus.

$3-$

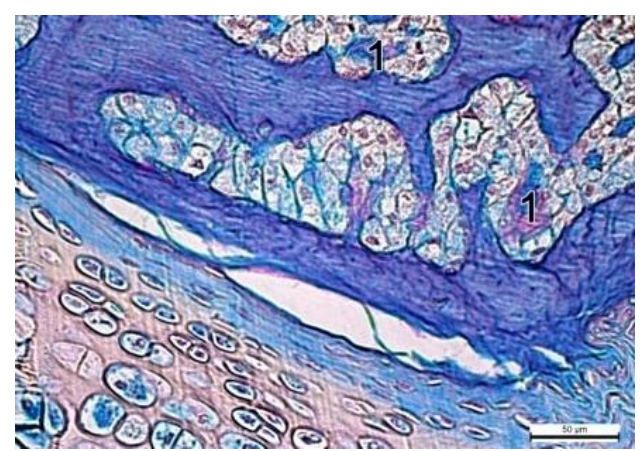

Figure 8. Intervertebral disc after 7 days of opioid use. Staining: alcian blue after Steedman. Photomicrograph x 400 .

1- inhomogeneous arrangement of acidic glycosaminoglycans in the matrix of the nucleus pulposus.

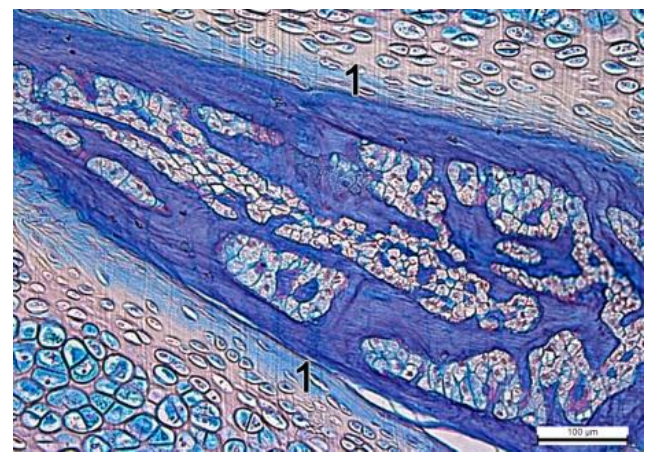

Figure. 9. Intervertebral disc after 7 days of opioid use. Staining: Alcian blue after Steedman. Photomicrograph x 200.

1- decrease in the content of acidic glycosaminoglycans in the collagen bundles of the annulus fibrosus.

Inclusions of acidic glycosaminoglycans were visualized locally. Accumulation of acidic glycosaminoglycan grains was observed near dystrophically altered notochordal cells. 
The content of acidic and primary glycosaminoglycans in the collagen bundles of the annulus fibrosus was decreased. However, in some swollen collagen fibers of the outer zone of the annulus fibrosus, the content of acidic glycosaminoglycans increased inhomogeneously (Figure 10).

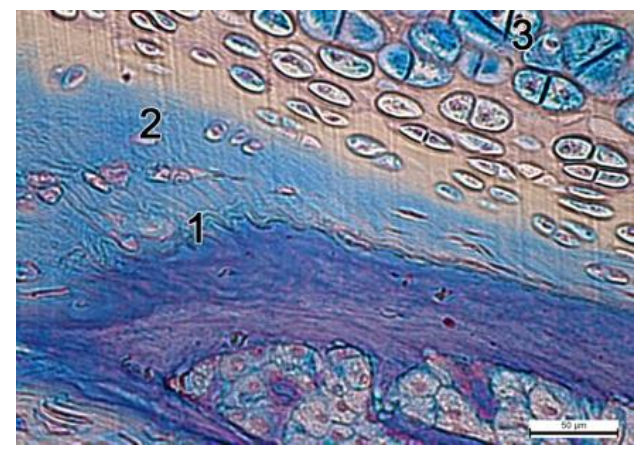

Figure 10. Intervertebral disc after 7 days of opioid use. Staining: Alcian blue after Steedman. Photomicrograph x 200.

1- decrease in the content of acidic glycosaminoglycans in the collagen bundles of the annulus fibrosus.

2- increase in the content of acidic glycosaminoglycans in the swollen collagen fibers of the outer zone of the annulus fibrosus;

3- increase in the content of acidic glycosaminoglycans in dystrophically altered chondrocytes of the endplates.

After 14 days of the opioid use at a dose of $15 \mathrm{mg} / \mathrm{kg}$ at we found alternative changes in notochordal cells of the nucleus pulposus, as well as destruction of collagen fibers of the annulus fibrosus and cartilage. The development of necrotic changes in notochordal cells, accompanied by karyopyknosis, was found in reduced nucleus pulposus (the nuclei of notochordal cells were reduced, intensely basophilic). Due to the development of necrotic changes, the number of notochordal cells in the nucleus pulposus decreased (Figure 11).

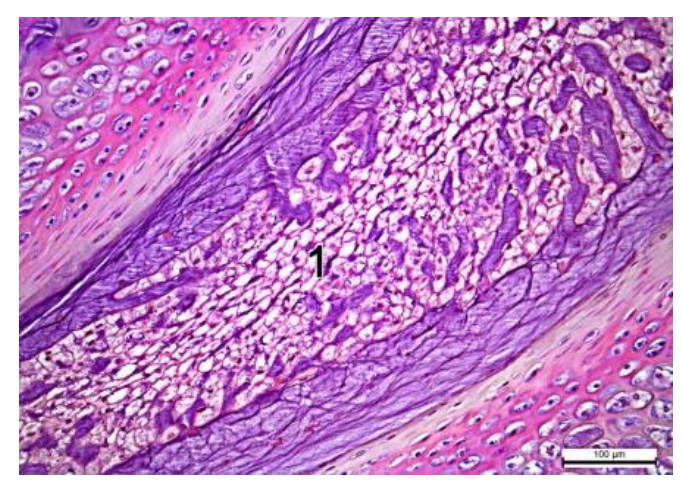

Figure. 11. Intervertebral disc after 14 days of opioid use. Hematoxylin and eosin staining. Photomicrograph x 200.

1- decrease in the number of notochordal cells in the nucleus pulposus. 
Preserved notochordal cells were located isolated. Cell-free zones appeared. The primary glycosaminoglycans were located in the matrix of the nucleus pulposus inhomogeneously, their content in the most areas was decreased (Figure. 12). The content of acidic glycosaminoglycans was increased in the areas of significant necrotic changes of notochordal cells. In cell-free zones intercellular matrix of the nucleus pulposus, dusty inclusions of acidic glycosaminoglycans accumulated.

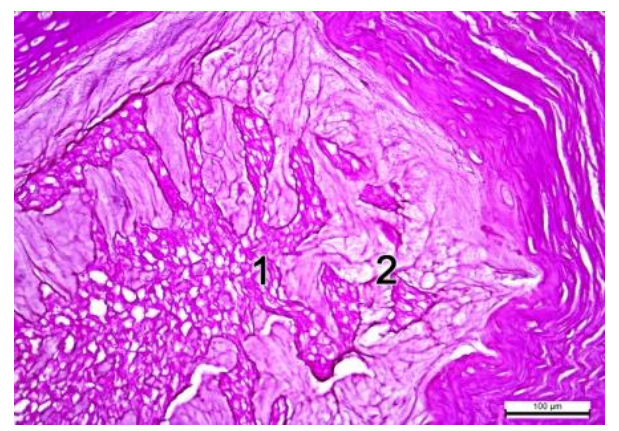

Figure 12. Intervertebral disc after 14 days of opioid use. Staining: PAS-reaction after McManus. Photomicrograph x 200.

1- decrease in the content of primary glycosaminoglycans in collagen bundles;

2- decrease in the content of primary glycosaminoglycans in the stroma of the nucleus pulposus.

The collagen bundles of the inner zone of the annulus fibrosus were arranged in a disordered manner, some of them acquired an irregular shape and flattened. Collagen fibers in the inner and outer zone of the annulus fibrosus were inhomogeneously swollen (Figure 13), they had slightly increased content of acidic glycosaminoglycans. At the same time, the content of primary glycosaminoglycans in the collagen bundles of the annulus fibrosus was decreased (Figure 14).

Due to the dissection of collagen fibers in the outer zone of the annulus fibrosus longitudinal cavities were formed, (Figure 14) in the walls of which the number of acidic glycosaminoglycans was slightly increased. 


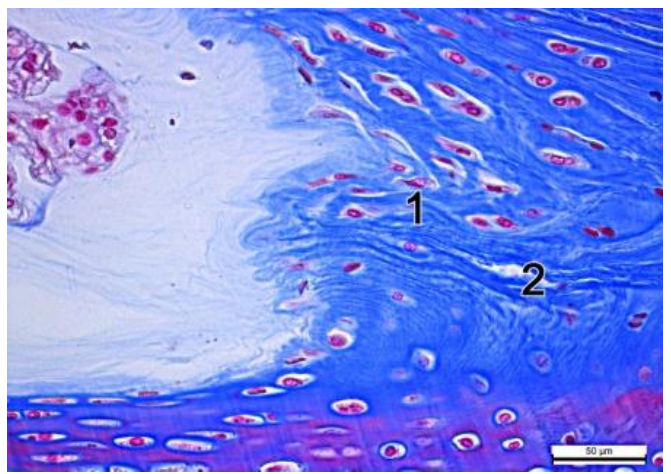

Figure 13. Intervertebral disc after 14 days of opioid use. Staining: Alcian blue after Steedman. Photomicrograph x 400.

1- inhomogeneous swelling of collagen fibers of the annulus fibrosus;

2- dissection of collagen fibers of the annulus fibrosus.

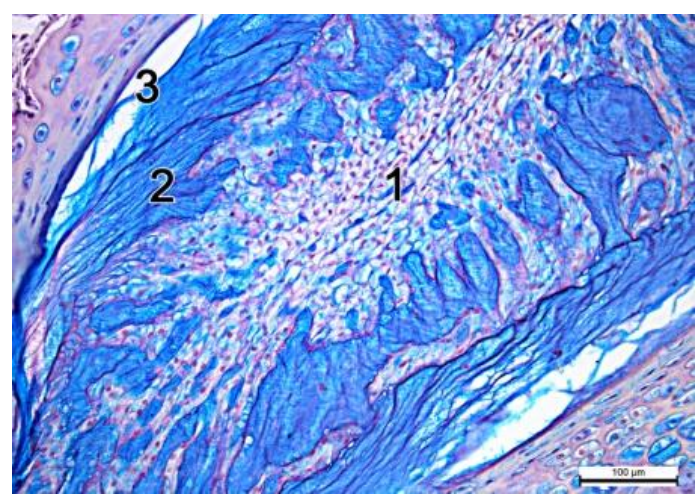

Fig. 14. Intervertebral disc after 14 days of opioid use. Staining: Alcian blue after Steedman. Photomicrograph x 200.

1- reduction in the content of acidic glycosaminoglycans in the collagen bundles of the annulus fibrosus;

2- slight increase in the content of acidic glycosaminoglycans in the swollen collagen fibers of the outer zone of the annulus fibrosus;

3- dissection of collagen fibers from the cartilage endplates.

Dystrophic and necrotic changes of chondrocytic endplates were also found (Figure 15). In dystrophically altered chondrocytes, the cytoplasm was swollen, containing volume vacuoles filled with optically clear cytoplasmic fluid. Under conditions of necrotic changes development of pyknotic nuclei in chondrocytes' cytoplasm were visualized. The content of acidic glycosaminoglycans was increased in the inner wall of the gaps and in dystrophically altered chondrocytes. 


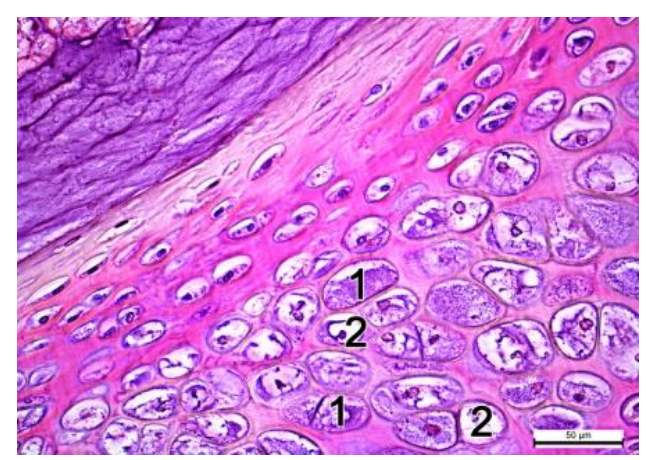

Figure 15. Intervertebral disc after 14 days of opioid use. Hematoxylin and eosin staining. Photomicrograph x 400 .

1- dystrophic changes of chondrocytes;

2- necrotic changes of chondrocytes.

Future directions: Pathomorphological features in the structure of the intervertebral disc at the end of the $7^{\text {th }}$ and $14^{\text {th }}$ days of opioid use will provide the opportunity to create a morphological basis for understanding of the smoothness and growth dynamics of pathomorphological changes in the structure of intervertebral discs. Our results will provide the opportunity to conduct a comparative analysis of pathomorphological changes in the structure of the intervertebral disc in the early and late stages of experimental opioid use.

\section{Conclusions:}

1. At the microstructural level we found the initial changes such as moderate degeneration in notochordal cells of nucleus pulposus and dissection of collagen fibers in annulus fibrosus after 7 days of opiod use. Besides, the development of dystrophic changes with swelling, enlightenment and vacuolation of the cytoplasm was observed in the chondrocytes of cartilage endplates.

2. After 14 days of an experiment we found alternative changes in notochordal cells of the nucleus pulposus, as well as destruction of collagen fibers of the annulus fibrosus and cartilage of the intervertebral disc. The development of necrotic changes in notochordal cells, including karyopyknosis, was found in a reduced nucleus pulposus. Due to the development of necrotic changes, the number of notochordal cells in the pulposus nucleus was decreased.

3. As a result of the dissection of collagen fibers in the outer zone of the annulus fibrosus between the cartilage endplates and the annulus fibrosus, longitudinal cavities were formed. Dystrophic and necrotic changes of chondrocytes of endbundles were also revealed. 


\section{References}

1. Biriuchkov MIu. The specific features of surgery of herniated intervertebral disks. Zh Vopr Neirokhir Im N N Burdenko. 2005 Oct-Dec; 4:22- 4.

2. Pinchuk SV. Modeling the lumbar spine computer tomography sizes on median-sagittal sections using regression analysis in healthy young men depending on anthropo-somatotypological indicators. Pinchuk SV, Volkov KS, Kryvko YuYa., Shayuk AV. Aktualni pytannya medychnoi nauky ta praktyky. 2015; 82. P. 136-42.

3. Pinchuk SV, Shayuk AV. A clinical value and problems of determination of morphometric parameters of lumbar spine in a norm and at pathology. Review of literature. Ukrainskyi medychnyi almanakh. 2013;16.5. P.101-106.

4. Gubin AV. The etiology of acute torticollis in children. In: Proceedings of the scientific-practical conference with international participation. Gubin AV, Ulrich EV, Yalfimov AN, Taschilkin AI. Actual problems of children's traumatology and orthope- dics; 2008; Kazan, Russia. Kazan; 2008. P.170-171.

5. Dudin MG. On etiopathogenesis of idiopathic scoliosis. Dudin MG, Pinchuk DYu, Bekshaev SS, et.all. Spine Surgery. 2006;(4):18-25.

6. Dmitriev AE, Tenants VK, Vavilov SB. Computed tomography in the diagnosis of degener- ative lesions of the spine - intervertebral disc herniation. Clinical Medicine. 2009. 4. P. 93-95.

7. Kostyuk GY, Vlasyuk VL Magnetic resonance imaging in the diagnosis and treatment of herniated discs. Clinical anatomy and operative surgery. 2008;7. 3. P. 53-56.

8. Hosten N. Computed tomography of the head and spine. 2nd ed. Hosten N, Liebig T. MED press-inform; 2013. 576 p.

9. Onysko RM, Paltov EV, Fik VB, Vilkhova IV, Kryvko Yu.Ya. ., Yakymiv N.Ya., Fitkalo OS. Pat. №76564 U Ukraine, IPC A 61 K 31/00 Method of modeling physical opioid dependence in rats. Patent owner: Danylo Halytskyi Lviv National Medical University. 201u201207124; declared 12.06.2012; publ. 10.01.2013, Bull. №1.

10. Romeis B. Microscopic technique. M. Medicine. 1953: 71 - 72.

11. Goralsky LP, Khomich VT, Kononsky OI Edited by Goralsky LP Fundamentals of histological technique and morphofunctional research methods in normal and pathology. Tutorial. Kind. III, corrected and supplemented. Zhytomyr: Polissya.2015:286. 